\title{
IL DIRITTO ALLA SALUTE DEGLI STRANIERI: STRUMENTI DI TUTELA ALLA LUCE DELLA NORMATIVA NAZIONALE ED INTERNAZIONALE
}

\section{Dott.ssa Allocca Roberta}

Dottoranda di ricerca in "Il benessere lavorativo della persona tra diritto, educazione e sviluppo sociale. - XXXIV Ciclo - A.A. 2018/19"

KEYWORDS: Diritto alla salute - Testo Unico sull'Immigrazione - D.1. 113/2018 - Permesso di soggiorno per cure mediche

\section{ABSTRACT}

Il presente lavoro vuole affrontare ed analizzare il tema del diritto alla salute degli stranieri, trattato alla luce delle convenzioni internazionali che ne regolamentano gli aspetti salienti, e dell'evoluzione normativa nazionale che definisce l'insieme degli obblighi e delle procedure previsti in Italia per poter esercitare tale diritto, in un percorso analitico trasversale che delinea un assetto normativo multidimensionale.

Si vuole tentare di fornire uno sguardo ragionato sulla condizione del diritto alla salute della popolazione immigrata presente nel nostro Paese, sugli strumenti che tutelano il diritto alla salute di queste persone e le modalità con cui vengono applicati nella prassi operativa.

\section{INTRODUZIONE}

L'immigrazione rappresenta per l'Italia un'importante occasione per riflettere sull'efficacia in senso universale dei principi del costituzionalismo italiano individuati quali capisaldi del nostro ordinamento circa settant'anni fa nella Costituzione repubblicana del 1948.

La presenza di cittadini stranieri che vivono e risiedono, spesso stabilmente, nel territorio statale mette alla prova i principi di inviolabilità dei diritti umani, di solidarietà e di eguaglianza, sanciti nei primi articoli della Costituzione in quanto principi fondamentali della Repubblica italiana.

Sembra necessario innanzitutto chiarire se i diritti fondamentali tutelati dalla Costituzione abbiano come destinatari soltanto i cittadini italiani o comprendano anche gli stranieri. A questo proposito una prima risposta da parte della Corte costituzionale è arrivata già alla fine degli anni Sessanta con le sentt. n. 120/1967 e n. 104/1969, nelle quali essa ha riconosciuto come applicabile anche agli stranieri il principio di eguaglianza per ciò che attiene alla tutela dei diritti inviolabili.

Di recente, essendo ormai chiaro che l'immigrazione verso l'Italia costituisce un fenomeno che ha raggiunto una certa stabilità strutturale nell'ordinamento, la Corte costituzionale ha affermato in modo più esplicito che «lo straniero è titolare di tutti i diritti fondamentali che la Costituzione riconosce spettanti alla persona», che «la condizione giuridica dello straniero non deve essere pertanto considerata - per quanto riguarda la tutela di tali diritti - come causa ammissibile di trattamenti diversificati e peggiorativi», e che per giustificare «l'ineluttabilità della limitazione di un diritto fondamentale» di uno straniero deve pertanto ravvisarsi l'esigenza di soddisfare un inderogabile interesse pubblico di rango costituzionale ${ }^{1}$.

In particolare, la tutela dei diritti fondamentali deve trovare un bilanciamento ragionevole con il controllo giuridico dell'immigrazione, al quale la Corte costituzionale ha appunto riconosciuto il valore di «interesse pubblico primario costituzionalmente rilevante» ${ }^{2}$.

A questo proposito il giudice costituzionale ha affermato che è possibile «subordinare, non irragionevolmente, l'erogazione di determinate prestazioni - non inerenti a rimediare a gravi situazioni di urgenza - alla circostanza che il titolo di legittimazione dello straniero al soggiorno nel territorio dello Stato ne dimostri il carattere non episodico e di non breve durata; una volta, però, che il diritto a soggiornare alle condizioni predette non sia in discussione, non si possono discriminare gli stranieri, stabilendo, nei loro confronti, particolari limitazioni per il godimento dei diritti fondamentali della persona, riconosciuti invece ai cittadini» ${ }^{3}$.

$\mathrm{Se}$, quindi, da un lato i più essenziali bisogni della persona non possono in nessun caso soccombere di fronte alle esigenze derivanti dal controllo dell'immigrazione, la Corte costituzionale ha però riconosciuto come non contrario alla Costituzione il bilanciamento operato dal legislatore tra l'accesso degli stranieri alle misure di tipo socio-assistenziale e la regolarità e la durata della loro presenza in Italia.

Per quanto riguarda l'assistenza sociale si può osservare come risulti ancora in via di definizione l'accesso dei cittadini di Paesi terzi ad alcune prestazioni socio-assistenziali erogate dallo Stato e dalle Regioni, come si evince, per esempio, dal filone giurisprudenziale riguardante la c.d. residenza protratta nel territo-

\footnotetext{
${ }^{1}$ Sentt. n. 105/2001 e n. 249/2010.

${ }^{2}$ Sent. n. 250/2010.

${ }^{3}$ Sentt. n. $432 / 2005$ e n. $148 / 2008$.
} 
rio nazionale/regionale ai fini dell'accesso ad alcune prestazioni o benefici di carattere sociale. Inoltre, l'accesso ai servizi socio-assistenziali risulta, in generale, strettamente dipendente dal requisito della residenza anagrafica che, a sua volta, presuppone la condizione di regolarità dello straniero che si trovi in Italia.

Le prestazioni di assistenza sociale che rispondono ai più essenziali bisogni della persona vanno però riconosciute a tutti, compresi gli stranieri privi di un valido titolo di soggiorno nel territorio italiano, come conseguenza dell'inderogabilità del dovere di solidarietà sociale previsto dall'art. 2 Cost.

Nel presente scritto si proverà a fare il punto sulle fonti che nell'ordinamento internazionale ed italiano garantiscono l'accesso degli stranieri all'assistenza sanitaria e sulle norme di dettaglio che disciplinano l'esercizio di tale diritto.

\section{METODOLOGIA E MATERIALI}

\section{Interventi giuridici di diritto internazionale e comunitario a tutela della salute}

Il diritto alla salute costituisce attualmente un obbligo legale internazionale degli Stati, ai quali è attribuito il compito di promuovere e tutelare la salute della propria popolazione. La prima comparsa dell'enunciazione del diritto universale e fondamentale alla salute in uno strumento di diritto internazionale in epoca moderna avvenne con la Dichiarazione Universale dei Diritti dell'Uomo del 1948 dove si rinviene il principio espressamente sancito dall'art. 25 secondo cui: «Ogni persona ha il diritto ad un adeguato livello di vita che assicuri a lui e alla sua famiglia la salute e il benessere, inclusi il cibo, il vestiario, l'abitazione, l'assistenza medica e i servizi sociali necessari, e il diritto alla sicurezza in caso di disoccupazione, malattia, disabilità, vedovanza e vecchiaia». L'anno successivo l'Organizzazione Mondiale della Sanità (OMS) nel suo stesso Atto costitutivo determinò la seguente definizione di salute: «La salute è uno stato di completo benessere fisico, mentale e sociale e non semplicemente un'assenza di malattia o infermità»; tale definizione, destinata a rovesciare completamente il dualismo concettuale salute-malattia, tende a scardinare la concezione del bisogno di salute inteso come bisogno di mera assistenza sanitaria, attribuendolo al contesto più ampio di vita sociale dell'individuo. Si intende tutelare dunque lo stato di benessere dell'individuo, non più una situazione di assenza di malattia, attraverso interventi che siano preventivi, curativi o riabilitativi. La definizione di salute dell'OMS pone altresì in evidenza la necessità di garantire l'equità nel processo di salvaguardia della salute: «Il diritto alla salute non deve essere concepito in termini di uguaglianza, bensì di equità. La non equità nella salute si riferisce alle differenze non necessarie ed evitabili e che sono, allo stesso tempo, inaccettabili e ingiuste. Equità sarà dunque favorire, per ciascun individuo, le condizioni per raggiungere il livello potenziale di salute che gli è proprio».

Il diritto alla salute in questo senso inteso verrà riconfermato e ampliato in uno dei fondamentali documenti dell'OMS, ovvero nella Dichiarazione di Alma Ata del 1978. Nella località di Alma Ata (all'epoca capitale della repubblica sovietica del Kazakhstan) si tenne in quell'anno la Conferenza Internazionale sull'Assistenza Sanitaria di Base (PrimaryHealth Care - PHC) promossa dall'OMS e dall'UNICEF. Fu un'occasione di incontro tra i rappresentanti del campo sanitario di tutti i Paesi del mondo e fu anche la prima volta nella storia che la questione dell'assistenza sanitaria di base e dei ruoli relativi di governi e organizzazioni internazionali veniva affrontata nell'ottica di collegare il tema della salute con quello dello sviluppo. Per la prima volta, infatti, si cercò di delineare dei principi di riferimento comuni per la promozione e lo sviluppo di un'assistenza sanitaria onnicomprensiva e universale e, inoltre, di trattare il problema sanitario che affliggeva i Paesi più poveri del mondo, pesantemente provati da lunghi periodi di oppressione coloniale. Risultato di questo percorso dialogico fu la stesura di una Dichiarazione che mirava a definire cosa si intendesse per PHC al fine di indirizzare le scelte politiche verso uno sviluppo efficiente e paritario dei sistemi sanitari del mondo ${ }^{4}$.

Ancora, il diritto alla salute è sancito agli artt. $10 \mathrm{e}$ 12 della Convenzione OIL n. 143 del 24 giugno 1975; all'art. 12 della Convenzione sull'eliminazione di tutte le forme di discriminazione contro le donne, adottata dall'Assemblea Generale delle Nazioni Unite nel 1979; e all'art. 24 della Convenzione sui diritti dell'infanzia, dell'Assemblea Generale delle Nazioni Unite del 20 novembre 1989.

Nel campo della bioetica i medesimi principi permearono la nascita del Belmont Report nel 1979, avente il compito di identificare i principi etici che avrebbero dovuto guidare la condotta della ricerca biomedica con soggetti umani, individuati in: rispetto per le persone (sia per la loro dignità che per la loro autonomia), la beneficenza (nel senso di beneficio apportato al paziente da parte del medico che in base alla sua esperienza conosce il bene oggettivo del paziente) e la giustizia ${ }^{5}$, la quale tende al bene comune nell'ottica appunto di una salute pubblica equamente distribuita nel limite delle risorse disponibili. Vanno citati infine l'art. 12 del Patto sui diritti economici, sociali e culturali, e l'art. 35 della Carta dei diritti fondamentali dell'Unione Europea, per il quale “ogni individuo

${ }^{4}$ «L'assistenza sanitaria di base è quell'assistenza sanitaria essenziale fondata su metodi e tecnologie pratiche, scientificamente valide e socialmente accettabili, resa universalmente accessibile agli individui e alle famiglie di una comunità attraverso la loro piena partecipazione a un costo che la comunità e i Paesi possono permettersi ad ogni stadio del loro sviluppo in uno spirito di fiducia in sé stessi e di autodeterminazione. Essa è parte integrante sia del Sistema Sanitario nazionale, di cui è il perno e il punto focale, che dello sviluppo economico e sociale globale della comunità. [...] Essa include almeno: l'educazione riguardo i più importanti problemi sanitari e i metodi per prevenirli e controllarli; la promozione di un'adeguata alimentazione; un'adeguata disponibilità di acqua potabile e condizioni salubri di vita; l'assistenza materno - infantile, inclusa la pianificazione familiare; le vaccinazioni contro le maggiori malattie infettive; la prevenzione e il controllo delle malattie endemiche; l'appropriato trattamento delle comuni malattie e l'approvvigionamento dei farmaci essenziali. [...] Coinvolge oltre al settore sanitario, tutti i settori e gli aspetti correlati allo sviluppo nazionale e della comunità, in particolare l'agricoltura, l'allevamento, l'alimentazione, l'industria, l'educazione, la casa, i lavori pubblici, le comunicazioni, e altri settori; e richiede lo sforzo coordinato di tutti questi settori». 
ha il diritto di accedere alla prevenzione sanitaria e di ottenere cure mediche alle condizioni stabilite dalle legislazioni e prassi nazionali”.

\section{La tutela del diritto alla salute: gli strumenti introdotti dalla normativa nazionale}

Spostando l'analisi sul piano nazionale, il diritto alla salute si presenta nel sistema costituzionale italiano quale diritto sociale fondamentale; ai sensi dell'art. 32 della Costituzione, infatti, "La Repubblica tutela la salute come fondamentale diritto dell'individuo e interesse della collettività, e garantisce cure gratuite agli indigenti". ${ }^{6}$

La dimensione pubblica e la portata generale della tutela della salute fanno sì che tale disposizione riguardi tutti gli individui e non solo i cittadini; essa si riconnette al contenuto dell'art. 25 della Dichiarazione Universale dei Diritti dell'uomo e del cittadino che fa espresso riferimento alla tutela della salute proprio per ciascun individuo.

Sullo sfondo del principio solennemente dichiarato dalla Costituzione, il legislatore italiano ha approntato una serie di strumenti normativi volti a disciplinare l'attuazione del diritto alla salute nei confronti dello straniero.

Il primo di tali strumenti - che costituisce la base normativa tuttora vigente - è il d.lgs. 25 luglio 1998, n. 286, intitolato "Testo Unico delle disposizioni concernenti la disciplina della immigrazione e norme sulla condizione dello straniero" i cui artt. 34 e 35 pongono una netta distinzione tra straniero regolare e straniero irregolare.

Gli stranieri che soggiornano regolarmente sul territorio nazionale e che sono lavoratori, insieme ai loro familiari, godono di parità di trattamento e piena uguaglianza di diritti e doveri con i cittadini italiani e sono iscritti nelle liste del Servizio sanitario nazionale. È fondamentale precisare che l'iscrizione nelle liste del S.S.N. costituisce un obbligo per lo straniero e da tale obbligo derivano i diritti all'assistenza sanitaria uguali a quelli dei cittadini italiani e dell'Ue.

Se lo straniero è regolarmente soggiornante, ma non svolge attività lavorativa (ad es. studente, turista, ecc.) ha facoltà di iscriversi al S.S.N. ma non l'obbligo e può, in alternativa, stipulare una polizza assicurativa 7; se non stipula polizze assicurative e non si iscrive al S.S.N. potrà, comunque ricorrere alle prestazioni erogate dal S.S.N. secondo le modalità previste dai primi due commi dell'art. 35 T.U.I.

$\mathrm{Al}$ terzo comma, tale articolo prende in considerazione il caso degli stranieri non in regola con le dispo- sizioni che disciplinano l'ingresso ed il soggiorno nel territorio nazionale.

Naturalmente, quando si parla di stranieri si fa riferimento ai cittadini extracomunitari, provenienti, cioè, dai Paesi che non fanno parte del' Ue o che non abbiano particolari tipi di accordi con la Ue.

Allo straniero sono garantite, nei presidi pubblici ed in quelli privati accreditati, le cure ambulatoriali ed ospedaliere urgenti e comunque essenziali, ancorché continuative, per malattie ed infortuni, e sono estesi i programmi di medicina preventiva a salvaguardia della salute individuale e collettiva.

Appare dunque evidente l'incidenza della distinzione tra straniero regolare e irregolare sulla fruibilità delle cure erogate dal Servizio sanitario nazionale.

Allo straniero irregolare non è, infatti, concessa la facoltà di iscriversi al S.S.N. e possono essergli apprestate esclusivamente le "cure urgenti o comunque essenziali”.

Bisogna ricordare, tuttavia, che nell'ordinamento italiano la materia della salute è oggetto di legislazione concorrente tra Stato e Regioni.

Questo fa sì che la legislazione statale fissi principi di carattere generale, in relazione ai quali le leggi regionali dettano la disciplina di dettaglio, e proprio la legislazione regionale, in alcuni casi, è andata oltre i criteri generali stabiliti dalla legislazione statale.

Un esempio a riguardo ci viene fornito dalla legge regionale della Toscana, 9 giugno 2009, n. 29, la quale all'art. 6 co. 35 ha stabilito che "tutte le persone dimoranti nel territorio regionale, anche se prive di titolo di soggiorno, possono fruire degli interventi socio-assistenziali urgenti e indifferibili, necessari per garantire il rispetto dei diritti fondamentali riconosciuti ad ogni persona in base alla Costituzione ed alle norme internazionali".

In tal modo la Regione Toscana ha esteso l'ambito di applicazione della tutela della salute nei confronti degli stranieri non regolari.

A suo tempo il Governo centrale ha impugnato tale disposizione dinanzi alla Corte Costituzionale, ritenendo che lo straniero irregolare divenisse titolare di prestazioni aventi il carattere dell'urgenza e della indifferibilità in contrasto con la normativa dello Stato a cui compete la fissazione dei principi di carattere generale.

Nel caso specifico la Corte Costituzionale si è pronunciata in senso favorevole alla Regione Toscana ritenendo legittima l'estensione del diritto alla salute dello straniero non regolare ed affermando che l'equiparazione attuata dalla legge regionale Toscana

\footnotetext{
${ }^{6}$ Sulla qualificazione del diritto alla salute quale diritto fondamentale, cfr., tra i contributi autorevoli più recenti, D. Morana, La salute come diritto costituzionale, Giappichelli, Torino, 2013; R. Balduzzi e D. Severetti, La garanzia costituzionale del diritto alla salute e la sua attuazione nel Serviziosanitario nazionale, in R. Balduzzi, G. Carpani (a cura di), Manuale di diritto sanitario, Il Mulino, Bologna 2013, pp. 25 ss.; D. Messineo, La garanzia del contenuto essenziale dei diritti fondamentali.Dalla tutela della dignità umana ai livelli essenziali delle prestazioni, Giappichelli, Torino 2012; $\mathrm{P}$. Grossi, Diritti fondamentali e diritti inviolabili nella Costituzione italiana, in Il diritto costituzionale tra principi di libertà e istituzioni, Cedam, Padova, 2008, p. 2 ss.

${ }^{7}$ Evidentemente, la presenza in Italia per motivi di lavoro è presumibilmente durevole, il che implica l'obbligo di assicurazione al S.s.n. Negli altri casi (turismo, studio, ecc.) in cui il soggiorno è più o meno breve, è lasciata alla scelta dello straniero la possibilità se iscriversi o meno al S.s.n. o stipulare una polizza privata, o se del caso, ricorrere al S.s.n. nei termini previsti dall' art. 35 T.U.I., co. 1 e 2, sul punto, cfr., G. Vosa, Cure essenziali. Sul diritto alla salute dello straniero irregolare: dall'auto determinazione della persona al policentrismo decisionale, in Dir. pubbl. 2/2016, p. 745; A. Romano, Salute e immigrazione. Prospettive di osservazione nella relazione tra l' accesso alle cure del non cittadino e l'organizzazione sanitaria, in M. Sesta (a cura di) L'erogazione della prestazione medica tra diritto alla salute, principio di autodeterminazione e gestione ottimale delle risorse sanitarie, Rimini,Maggioli, 2014, pp. 69 ss.
} 
fra stranieri regolari e irregolari non contrasta con i principi costituzionali in materia. ${ }^{8}$

Giova precisare, altresì, che già in precedenza la Corte Costituzionale si era espressa nel senso di ritenere che, poiché i diritti fondamentali sono riconosciuti alla persona intesa come tale, non possono essere ammesse discriminazioni tra cittadini e stranieri ${ }^{9}$.

La forza espansiva di tale principio fa sì che il cittadino straniero anche se irregolare debba essere considerato quale persona titolare, al pari delle altre (cioè cittadini e stranieri regolari), dei diritti fondamentali ${ }^{10}$

Tuttavia, alla estensione della tutela sanitaria dello straniero irregolare si sono contrapposte negli ultimi anni due vicende riguardanti, rispettivamente, il condizionamento economico - finanziario dei diritti fondamentali, ed in particolare di quello alla salute, e la crescente dimensione del fenomeno immigratorio irregolare.

Sotto il primo aspetto, il diritto alla salute si pone come diritto sociale finanziariamente condizionato, che trova limiti oggettivi in relazione alle risorse organizzative e finanziarie di cui dispone il nostro Paese.

La Corte Costituzionale si è resa interprete di questa esigenza affermando che a causa della limitatezza delle risorse finanziare, è necessario commisurare la spesa sanitaria alle effettive disponibilità finanziarie.

La stessa Corte si è preoccupata, altresì, di individuare un limite invalicabile a fronte del quale l'esigenza di mantenere l'equilibrio della finanza pubblica deve venire meno rispetto al peso preponderante della tutela della salute; tale limite si rinviene nel nucleo essenziale del diritto alla salute connesso alla inviolabile dignità della persona umana.

Sulla base anche di dette pronunce, la legislazione statale si è orientata in tale direzione ${ }^{11}$.

La stessa mobilità sanitaria interna nel territorio nazionale italiano subisce limitazioni in relazione alle esigenze di carattere economico - finanziario, specialmente per quanto riguarda i rimborsi e le compensazioni tra le Regioni, sulle quali grava la gestione degli apparati sanitari.

Il secondo aspetto, inevitabilmente collegato al primo, è quello relativo al crescente numero di stranieri ir- regolari dimoranti in Italia, a seguito di flussi immigratori quotidiani difficilmente controllabili, che vedono coinvolte centinaia di persone che sbarcano sulle coste italiane dopo essere fuggite da guerre, situazioni di grave pericolo o di miseria negli Stati di appartenenza da cui provengono.

Questo duplice ordine di fenomeni ha comportato, sia nella normativa che nella prassi, una profonda divaricazione nell' esercizio del diritto sociale alla salute tra il cittadino (italiano ed UE, al quale viene sostanzialmente assimilato lo straniero extracomunitario regolarmente soggiornante in Italia) e lo straniero irregolare.

Al primo è assicurata la pienezza dell'esercizio del diritto alla salute, al secondo è assicurata la prestazione "di cure ambulatoriali ed ospedaliere urgenti, e comunque essenziali, ancorché continuative".

Per rendersi conto di questa divaricazione occorre considerare che l'esercizio del diritto costituzionale alla salute si traduce per il cittadino (oltre ai diritti di libertà di come e da chi farsi curare o anche di non farsi curare) nel diritto a ottenere le prestazioni sanitarie nei termini e con le modalità fissati nei livelli essenziali di assistenza (i cd. Lea).

I Lea possono essere definiti come l'insieme delle prestazioni appropriate (sia sotto l'aspetto clinico che sotto l'aspetto economico) e necessarie a garantire la tutela della salute, e sono pertanto da intendersi non come un contenuto minimo, ma come un contenuto necessario, fissato con disposizioni normative ${ }^{12}$.

Ma proprio la diversa connotazione della essenzialità delle prestazioni sanitarie garantite dallo Stato a seconda che si tratti di cittadino (o ad esso assimilato) e straniero irregolare dà la misura della divaricazione attualmente esistente.

Nel primo caso l'essenzialità costituisce un contenuto al di sotto del quale non si può scendere; nel secondo caso l'essenzialità costituisce un contenuto al di sopra del quale non si può salire.

I Lea per il cittadino rappresentano la misura ritenuta sufficiente per garantire il diritto alla salute, intendendosi per salute non semplicemente l'assenza di malattie o infermità, ma una condizione di benessere fisico, mentale e sociale.

Per lo straniero irregolare le cure essenziali sono

${ }^{8}$ Sent. 7 luglio, 2010, n. 269.Per un commento alla sentenza, v. F. Biondi Dal Monte, Regioni, immigrazione e diritti fondamentali, in www.forumcostituzionale.it,2010, pp. 6 ss.

${ }^{9}$ In particolare, per quanto riguarda il diritto alla salute, cfr. Corte Cost. 5 luglio, 2001, n. 252, in forza della quale il diritto alla salute, protetto dalla Costituzione come ambito inviolabile della dignità umana, deve esserericonosciuto "anche agli stranieri, qualunque sia la loro posizione rispetto alle norme che regolano l'ingresso per il soggiorno nello Stato, pur potendo il legislatore prevedere diverse modalità di esercizio dello stesso".

${ }^{10}$ Secondo G. Vosa "Grazie all'elaborazione concettuale in materia di diritti, la Corte ha di fatto introdotto nell'ordinamento repubblicano corposi elementi di tutela delle posizioni afferenti allo straniero, ancorché irregolare. Tali elementi da un canto si riconducono all' intangibilità del contenuto essenziale dei diritti fondamentali; per altro verso, fanno capo al divieto di ingiustificata discriminazione, che affonda le proprie radici nel terreno del giudizio di ragionevolezza" (op. cit., p. 751).

${ }^{11}$ Sul punto cfr. F. G. Cuttaia, Il condizionamento finanziario del diritto sociale alla salute: fondamento e prospettive evolutive, in Astrid, 9/2017; G. Bertolissi, Il contenimento della spesa sanitaria tra innovazioni legislative e riscontri giurisdizionali, in Sanità pubblica e privata, luglio - sett. 2015, p. 57 ss.; L. Monteferrante, La dignità della persona umana tra istanze di tutela dei diritti sociali ed esigenze di contenimento della spesa pubblica: prospettive a confronto, in G.C. De Martin, D. Morana (a cura di) Amministrazione e democrazia, Cedam, Padova, 2013, p. 113 ss.

${ }^{12}$ Non possono che, in questa sede riassumersi i tratti fondamentalo dei lea, sulla cui natura e contenuto si rinvia bel dettaglio, ex multis, ai più recenti contributi di M. Atripaldi, Diritto alla salute e livelli essenziali di assistenza (LEA), in www.Fedarlismi.it, 15/11/2017; F.G. Cuttaia, Entrati in vigore i nuovi LEA, in Astrid, 21 marzo 2017; F. S. Florio, L'evoluzione dei LEA tra i recenti interventi normativi e le prospettive di riforma, in C. Bottari (a cura di), I livelli essenziali delle prestazioni sociali e sanitarie, Maggioli, Rimini, 2014, p. 53 ss. 
quelle indispensabili, di cui non si può fare a meno ${ }^{13}$.

Infatti, secondo la circolare 24 marzo 2000, n. 5 del Ministero della Salute esse sono quelle "che non possono essere differite senza pericolo per la vita e danno per la salute della persona" e sono essenziali "le prestazioni sanitarie, diagnostiche e terapeutiche relative a patologie non pericolose nell'immediato o nel breve termine, ma che nel tempo potrebbero determinare maggior danno alla salute o rischi per la vita". Quella che può essere definita come una divaricazione è comunque compatibile con il quadro costituzionale italiano, in quanto la tutela del nucleo essenziale del diritto alla salute dello straniero irregolare risponde al principio della inviolabilità dei diritti fondamentali dell'individuo, mentre il diverso contenuto da attribuire al livello essenziale della prestazione sanitaria, a seconda che si tratti di cittadino o straniero irregolare, appare in linea con il tenore dell'art. 10 Cost., in base al quale "l'ordinamento giuridico italiano si conforma alle norme del diritto internazionale generalmente riconosciute. La condizione giuridica dello straniero è regolata dalla legge in conformità delle norme e dei trattati internazionali".

In proposito, la Corte Costituzionale ha affermato che "non può escludersi che tra cittadino e straniero, benché uguali nella titolarità di certi diritti di libertà, esistano differenze di fatto che possano giustificare un loro diverso trattamento nel godimento di quegli stessi diritti", significando che tali differenze sono riconducibili alla "basilare differenza esistente tra il cittadino e lo straniero, consistente nella circostanza che, mentre il primo ha con lo Stato un rapporto di solito originario e comunque permanente, il secondo ne ha uno acquisito e generalmente temporaneo" 14 .

D' altra parte, come opportunamente è stato osservato in dottrina ${ }^{15}$, è ravvisabile una divaricazione nella stessa giurisprudenza costituzionale laddove, in generale, in ordine alla condizione giuridica dello straniero, la stessa Corte ammette che "al legislatore è consentito dettare norme, non palesemente irragionevoli, che regolino l'ingresso e la permanenza di extracomunitari in Italia, ma una volta che il diritto a soggiornare non sia in discussione, non si possono discriminare gli stranieri stabilendo nei loro confronti particolari limitazioni né per il godimento dei diritti fondamentali della persona, né nell' esercizio dei doveri di solidarietà previsti dalla Costituzione" ${ }^{\prime 16}$. Dal che deriva una sorta di equiparazione del cittadino straniero al cittadino italiano nella prestazione dei diritti sociali sulla base della valutazione, operata nei confronti del primo, circa la sussistenza del criterio del regolare soggiorno sul territorio nazionale.

Il trattamento differenziato, però, non si ha quando ci si trovi di fronte a stranieri minori non accompagnati.

Essi sono coloro che si trovano per qualsiasi causa nel territorio dello Stato, privi di assistenza e rappresentanza da parte di genitori o altri adulti legalmente responsabili ${ }^{17}$.

La 1.7 aprile 2017 , n. 47 ha introdotto una disciplina che, nel prevedere maggiori tutele rispetto al passato, ha stabilito la piena assistenza sanitaria per i minori stranieri non accompagnati. Riguardo ad essi è prevista l'iscrizione obbligatoria al S.S.N. ${ }^{18}$.

La disciplina introdotta dalla 1. n. 47/2017 appare improntata alla finalità di superare la più volte indicata divaricazione nella disciplina riservata rispettivamente agli stranieri regolarmente soggiornanti nel nostro Paese e a coloro che non lo sono, in una logica tendente a considerare il diritto alla salute come "l'unico diritto della persona che la Costituzione qualifica come fondamentale, quale nucleo fondativo di tutti gli altri diritti costituzionali e presupposto irrinunciabile per la piena realizzazione della persona umana" ${ }^{\prime 19} \mathrm{e}$ che probabilmente richiederebbe a livello di normativa Ue una più puntuale definizione per garantire alle persone di origine extracomunitaria una uniformità di trattamento.

Un ultimo aspetto da analizzare a proposito del diritto alla salute degli stranieri, per la sua attinenza con il d.l. n. 113/2018 entrato in vigore pochi mesi fa, è quello della misura entro cui la malattia può giustificare la permanenza in Italia dello straniero e, più nello specifico, rendere inefficace un decreto di espulsione dal territorio italiano.

Prendendo come riferimento i Lea 2017 di cui si è detto, allo straniero irregolare dovrebbe essere garantita la cura di qualsiasi patologia che possa comportare «un danno per la salute» o «un rischio per la vita»: da ciò si potrebbe ricavare la norma, molto ampia, secondo cui nessuno straniero potrebbe essere espulso dall'Italia fintanto che non sia accertata la sua comple-

\footnotetext{
${ }^{13}$ Secondo S. D' Antonio, in tal modo si pongono in evidenza due essenzialità diverse, una per i Lea e una per le cure dello straniero irregolare, venendosi cosi così a configurare una deminutionei confronti dello straniero irregolare, fondata esclusivamente su uno status personale (S. D' Antonio, Appunti introduttivi sul diritto alla salute degli stranieri nell' ordinamento italiano, in F. Rimoli (a cura di), immigrazione e integrazione. Dalla prospettiva globale alle realtà locali, Napoli, ES. 2014, vol. I, pp. 97 ss.
}

${ }^{14}$ Sent. 19 giugno, 1969, n. 104, il cui orientamento è stato confermato da successive pronunce, di recente con sent. 20 luglio, 2011, n. 245.

${ }^{15} \mathrm{Cfr}$., in particolare, M. Savino, Lo straniero nella giurisprudenza costituzionale: tra cittadinanza e territorialità, in Quaderni costituzionali, $1 / 2017$, p. 63 ss.

${ }^{16}$ Corte Cost. 10 dicembre 2013, n. 309.

${ }^{17}$ Per una valutazione delle cause e dei flussi migratori relativi ai minori stranieri non accompagnati, v. V. Melchionda, I minori stranieri non accompagnati in Italia, in Nuova etica pubblica, n. 9/2017, pp. 85 ss.

\footnotetext{
${ }^{18}$ Tuttavia, come è stato evidenziato in dottrina, tale legge, pur introducendo principi di rilevante importanza, è soggetta alla clausola della invarianza finanziaria, con il rischio che, l' esercizio del diritto alla salute sia condizionato dalla situazione economica (sul punto cfr. M. Gnes, Le nuove misure per la protezione dei minori non accompagnati, in Quotidianogiuridico.it, 2017); G. A. Giuffrè, Il regime di assistenza sanitaria dei minori stranieri non accompagnati alla luce della l. n. 47 del 2017, inFederalismi.it, 15 novembre 2017.
}

${ }^{19} \mathrm{G}$. Zagrebelsky, Prefazione a Sanità e diritti fond mentali in ambito europeo e italiano (a cura di) L.S. Rossi e C. Bottari, Maggioli, Rimini, 2013. 
ta guarigione da qualsiasi patologia che abbia le caratteristiche previste dall'art. 63 dei Lea 2017.

Finora, tuttavia, la Cassazione sembra avere accolto un'impostazione più restrittiva, giustificando la permanenza in Italia dello straniero irregolare soprattutto in caso di «prestazioni essenziali per la vita».

Anche la giurisprudenza amministrativa sembra avere assunto posizioni non molto distanti da quelle

dei giudici ordinari, avendo posto l'accento sulle cure "salva vita», sulla "cronicità della malattia», sull'«indifferibilità degli interventi» e sul fatto che «le cure per la patologia non potrebbero essere adeguatamente prestate nel Pese di origine», per giustificare l'annullamento di un provvedimento di espulsione dello straniero e dunque la sua permanenza in Italia $^{20}$.

Allo straniero irregolare, secondo quanto affermato dal Consiglio di Stato, può essere riconosciuto lo «status di non espellibilità» qualora necessiti di «interventi che, successivi alla somministrazione di farmaci essenziali per la vita, siano indispensabili al completamento dei primi o al conseguimento della loro efficacia, mentre restano esclusi quei trattamenti di mantenimento o di controllo che, seppur indispensabili ad assicurare una spes vitae per il paziente, fuoriescono dalla correlazione strumentale con l'efficacia immediata dell'intervento sanitario indifferibile e urgente».

Tuttavia, un tale stato di salute non giustificava sempre il rilascio di un permesso di soggiorno per cure mediche, peraltro non previsto dal T.U.I. prima dell'entrata in vigore del d.l. n. 113/2018.

Ciò nonostante, il permesso di soggiorno per cure mediche poteva essere ottenuto dallo straniero irregolare - adottando una procedura atipica che estende agli stranieri irregolari il permesso di soggiorno per cure mediche previsto dall'art. 36 T.U.I. soltanto per gli stranieri regolari - in presenza di «circostanze eccezionali e gravissime connesse allo stato di salute dell'interessato ${ }^{21}$.

Dunque, sia che allo straniero irregolare venisse riconosciuto in via giurisprudenziale lo status di «non espellibilità», sia che l'eccezionale gravità della sua malattia giustificasse il rilascio di un permesso di soggiorno per cure mediche, allo straniero malato non in regola con le condizioni di ingresso e di soggiorno è stata comunque assicurata fino ad oggi la permanenza in Italia per ricevere le cure urgenti ed essenziali, in modo non incompatibile con i principi che la Corte costituzionale ha affermato.

Il decreto "sicurezza" n. 113/2018, ha introdotto nell'art. 19, c. 2 , T.U.I. la lettera $d$-bis)disponendo che non possano essere allontanati o respinti «gli stranieri che versano in condizioni di salute di eccezionale gravità, accertate mediante idonea documentazione, tali da determinare un irreparabile pregiudizio alla salute degli stessi, in caso di rientro nel Paese di origine o di provenienza. In tali ipotesi, il questore rilascia un permesso di soggiorno per cure mediche, per il tempo attestato dalla certificazione sanitaria, comunque non superiore ad un anno, rinnovabile finché persistono le condizioni di salute di eccezionale gravità debitamente certificate, valido solo nel territorio nazionale». La legge di conversione ha in seguito modificato l'originaria formulazione dell'art. 19, c. 2, lett. d-bis) T.U.I. stabilendo che «gli stranieri che versano in condizio- ni di salute di particolare gravità, accertate mediante idonea documentazione rilasciata da una struttura sanitaria pubblica o da un medico convenzionato con il Servizio sanitario nazionale, tali da determinare un rilevante pregiudizio alla salute degli stessi, in caso di rientro nel Paese di origine o di provenienza. In tali ipotesi, il questore rilascia un permesso di soggiorno per cure mediche, per il tempo attestato dalla certificazione sanitaria, comunque non superiore ad un anno, rinnovabile finché persistono le condizioni di salute di particolare gravità debitamente certificate, valido solo nel territorio nazionale».

La nuova disposizione dell'art. 19, c. 2, lett. d-bis), sembra dare copertura legislativa alla giurisprudenza amministrativa di cui si è detto, e che aveva riconosciuto comunque la possibilità di rilasciare un permesso di soggiorno per cure mediche anche agli stranieri irregolari in presenza di «circostanze eccezionali e gravissime connesse allo stato di salute».

L'art. 19, c. 2, lett. d-bis, pertanto, non afferma la possibilità di rilasciare un permesso di soggiorno per cure mediche a tutti gli stranieri irregolari che necessitino di cure urgenti ed essenziali, ma soltanto a coloro che si trovino in "condizioni di salute di particolare gravità». Tuttavia non chiarisce se, una volta guarito, lo straniero sia comunque costretto a lasciare il territorio italiano oppure possa chiedere la conversione del permesso di soggiorno per cure mediche in un'altra tipologia di permesso di soggiorno (ad es. per motivi di lavoro).

\section{RISULTATI E DISCUSSIONE}

Il diritto alla salute degli stranieri emerge, dalle considerazioni appena concluse, quale diritto fondamentale sul quale si concentra un'attenzione giuridica espressa su più livelli, da quello internazionale e comunitario che ha provveduto a costruire un quadro di norme generali di riferimento, sino a quello nazionale che ha tentato di disciplinarne gli aspetti specifici relativi alle esigenze scaturite dalla realtà quotidiana del fenomeno immigratorio che interessa il nostro Paese.

Come si è cercato di dimostrare nel presente scritto, da sempre, sia prima, sia dopo l'istituzione del SSN da parte della legge n. 833/1978, l'ordinamento italiano ha riconosciuto agli stranieri una tutela ampia del diritto alla salute. Ciò grazie soprattutto all'art. 32 Cost., come interpretato dalla Corte costituzionale anche in rapporto ai cittadini di Paesi terzi comunque presenti in Italia e come concretamente attuato dal legislatore italiano. Dopo circa un ventennio, dal 1998 al 2018, in cui le norme che si occupano della tutela della salute degli stranieri sono state considerate parte integrante del diritto dell'immigrazione, con l'approvazione dei nuovi Lea 2017 tale disciplina è stata inoltre riportata nell'alveo del diritto sanitario. Questo dato sembra destinato a produrre degli effetti positivi per quanto riguarda il bilanciamento tra la tutela della salute degli stranieri - soprattutto irregolari - e l'interesse comunque costituzionalmente rilevante dello Stato al controllo dell'immigrazione. Ciò sembra inoltre dare luogo a una più corretta articolazione dei rapporti di competenza tra lo Stato e le Regioni previsto dall'art. 117 Cost. in materia di immigrazione, livelli essenziali di assistenza e tutela della salute.

\footnotetext{
${ }^{20}$ Cons. Stato, sez. III, sent. n. 5265/2018 del 6 settembre 2018.

${ }^{21}$ Cons. Stato, sez. III, sentt. 5265/2018, 5286/2011 e 3488/2014.
} 


\section{BIGLIOGRAFIA}

1. Atripaldi, Diritto alla salute e livelli essenziali di assistenza (LEA), in www.Fedarlismi.it, 15/11/2017;

2. Balduzzi e D. Severetti, La garanzia costituzionale del diritto alla salute e la sua attuazione nel Serviziosanitario nazionale, in R. Balduzzi, G. Carpani (a cura di), Manuale di diritto sanitario, Il Mulino, Bologna 2013, pp. 25 ss.;

3. Bertolissi, Il contenimento della spesa sanitaria tra innovazioni legislative e riscontri giurisdizionali, in Sanità pubblica e privata, luglio - sett. 2015, p. 57 ss.;

4. Biondi Dal Monte, Regioni, immigrazione e diritti fondamentali, in www.forumcostituzionale.it, 2010, pp. 6 ss.;

5. Cuttaia, Entrati in vigore i nuovi LEA, in Astrid, 21 marzo 2017;

6. Cuttaia, Il condizionamento finanziario del diritto sociale alla salute: fondamento e prospettive evolutive, in Astrid, 9/2017;

7. D' Antonio, Appunti introduttivi sul diritto alla salute degli stranieri nell'ordinamento italiano, in F. Rimoli (a cura di), immigrazione e integrazione. Dalla prospettiva globale alle realtà locali, Napoli, ES. 2014, vol. I, pp. 97 ss.;

8. Florio, L'evoluzione dei LEA tra i recenti interventi normativi e le prospettive di riforma, in C. Bottari (a cura di), I livelli essenziali delle prestazioni sociali e sanitarie, Maggioli, Rimini, 2014, p. 53 ss.;

9. Giuffrè, Il regime di assistenza sanitaria dei minori stranieri non accompagnati alla luce della l. $n .47$ del 2017, inFederalismi.it, 15 novembre 2017;

10. Gnes, Le nuove misure per la protezione dei minori non accompagnati, in Quotidianogiuridico.it, 2017);

11. Grossi, Diritti fondamentali e diritti inviolabili nella Costituzione italiana, in Il diritto costituzionale tra principi di libertà e istituzioni, Cedam, Padova, 2008, p. 2 ss.;

12. Melchionda, I minori stranieri non accompagnati in Italia, in Nuova etica pubblica, n. 9/2017, pp. 85 ss.;

13. Messineo, La garanzia del contenuto essenziale dei diritti fondamentali.Dalla tutela della dignità umana ai livelli essenziali delle prestazioni, Giappichelli, Torino 2012;

14. Monteferrante, La dignità della persona umana tra istanze di tutela dei diritti sociali ed esigenze di contenimento della spesa pubblica: prospettive a confronto, in G.C. De Martin, D. Morana (a cura di) Amministrazione e democrazia, Cedam, Padova, 2013, p. 113 ss.;

15. Morana, La salute come diritto costituzionale, Giappichelli, Torino, 2013;

16. Romano, Salute e immigrazione. Prospettive di osservazione nella relazione tra l'accesso alle cure del non cittadino e l'organizzazione sanitaria, in M. Sesta (a cura di) L'erogazione della prestazione medica tra diritto alla salute, principio di autodeterminazione e gestione ottimale delle risorse sanitarie, Rimini,Maggioli, 2014, pp. 69 ss.:

17. Savino, Lo straniero nella giurisprudenza costituzionale: tra cittadinanza e territorialità, in Quaderni costituzionali, $1 / 2017$, p. 63 ss.;

18. Turoldo, Bioetica e reciprocità, Roma, 2011;

19. Vosa, Cure essenziali. Sul diritto alla salute dello straniero irregolare: dall' auto determinazione della persona al policentrismo decisionale, in Dir. pubbl. 2/2016, p. 745;

20. Zagrebelsky, Prefazione a Sanità e diritti fondamentali in ambito europeo e italiano (a cura di) L.S. Rossi e C. Bottari, Maggioli, Rimini, 2013.

\section{RIFERIMENTI NORMATIVI}

1. Convenzione OIL n. 143,24 giugno 1975;

2. Convenzione sull'eliminazione di tutte le forme di discriminazione contro le donne, 1979;

3. Convenzione sui diritti dell'infanzia, 20 novembre 1989;

4. Cons. Stato, sez. III, sent. n. 5265/2018 del 6 settembre 2018;

5. Cons. Stato, sez. III, sentt. 5265/2018, 5286/2011 e 3488/2014;

6. Corte Cost. 10 dicembre 2013, n. 309;

7. Dichiarazione Universale dei Diritti dell'Uomo, 1948;

8. D.lgs. 25 luglio 1998 , n. 286, intitolato "Testo Unico delle disposizioni concernenti la disciplina della immigrazione e norme sulla condizione dello straniero"

9. D.1.n. 113/2018;

10. Sent. 19 giugno, 1969, n. 104;

11. Sentt. n. 105/2001 e n. 249/2010;

12. Sent. n. 250/2010;

13. Sentt. n. 432/2005 e n. $148 / 2008$;

14. Sent. 7 luglio, 2010, n. 269;

15. Sent. 20 luglio, 2011, n. 245. 